\title{
Voluntary and Reactive Recruitment of Locomotor Muscle Synergies during Perturbed Walking
}

\author{
Stacie A. Chvatal and Lena H. Ting \\ The Wallace H. Coulter Department of Biomedical Engineering at Georgia Tech and Emory University, Atlanta, Georgia 30322-0535
}

The modular control of muscles in groups, often referred to as muscle synergies, has been proposed to provide a motor repertoire of actions for the robust control of movement. However, it is not clear whether muscle synergies identified in one task are also recruited by different neural pathways subserving other motor behaviors. We tested the hypothesis that voluntary and reactive modifications to walking in humans result from the recruitment of locomotor muscle synergies. We recorded the activity of 16 muscles in the right leg as subjects walked a $7.5 \mathrm{~m}$ path at two different speeds. To elicit a second motor behavior, midway through the path we imposed ramp and hold translation perturbations of the support surface in each of four cardinal directions. Variations in the temporal recruitment of locomotor muscle synergies could account for cycle-by-cycle variations in muscle activity across strides. Locomotor muscle synergies were also recruited in atypical phases of gait, accounting for both anticipatory gait modifications before perturbations and reactive feedback responses to perturbations. Our findings are consistent with the idea that a common pool of spatially fixed locomotor muscle synergies can be recruited by different neural pathways, including the central pattern generator for walking, brainstem pathways for balance control, and cortical pathways mediating voluntary gait modifications. Together with electrophysiological studies, our work suggests that muscle synergies may provide a library of motor subtasks that can be flexibly recruited by parallel descending pathways to generate a variety of complex natural movements in the upper and lower limbs.

\section{Introduction}

The modular control of muscles in groups, often referred to as muscle synergies, has been demonstrated in both upper and lower limb tasks (d'Avella et al., 2006; Ting and McKay, 2007; Drew et al., 2008; Overduin et al., 2008). However, it remains unclear whether muscle synergies reflect a library of motor actions that can subserve multiple motor behaviors (Giszter et al., 2007; Ting and McKay, 2007; Tresch and Jarc, 2009; Roh et al., 2012). In locomotion and reaching, it has been suggested that hierarchical neural pathways act in parallel to recruit a common set of muscle synergies (Isa et al., 2007; Drew et al., 2008). Electrophysiological evidence in cats demonstrates that motor cortical neurons that are only active during voluntary modifications to gait (Drew, 1988) appear to recruit a set of muscle synergies that is also recruited by subcortical pathways during unobstructed locomotion (Drew et al., 2002). In frog, muscle synergies for various forms of locomotion in the intact animal are also recruited for behaviors elicited after decerebration or spinalization (Roh et al., 2011). Finally, in primate upper limb, motor cortical activity mediates voluntary movements as well as long-latency reactions to perturbations (Herter et al., 2009), possibly via com-

Received Dec. 20, 2011; revised July 6, 2012; accepted July 14, 2012.

Author contributions: S.A.C. and L.H.T. designed research; S.A.C. performed research; S.A.C. and L.H.T. analyzed data; S.A.C. and L.H.T. wrote the paper.

This work is supported by NIH Grant NS058322 to L.H.T., and NSF IGERT and NSF Graduate Research Fellowships to S.A.C. We thank Dr. Steven L. Wolf for his assistance in developing the experimental design.

Correspondence should be addressed to Lena H. Ting, The Wallace H. Coulter Department of Biomedical Engineering at Georgia Tech and Emory University, 313 Ferst Drive, Atlanta, GA 30332-0535. E-mail: Iting@emory.edu. DOI:10.1523/JNEUROSCI.6344-11.2012

Copyright $\odot 2012$ the authors $\quad 0270-6474 / 12 / 3212237-14 \$ 15.00 / 0$ mon interneurons in the spinal cord coordinating multiple muscles of the arm and hand (Dum and Strick, 1996; Takei and Seki, 2010; Alstermark and Isa, 2012).

Walking in natural environments involves the parallel activation of multiple neural pathways to coordinate locomotion, balance, and voluntary gait modifications. The basic locomotor pattern may be produced by spinal central pattern generators (Rossignol et al., 2006; McCrea and Rybak, 2008) which are modulated by sensory feedback pathways within the spinal cord (Forssberg et al., 1980; Rossignol et al., 2008). If discrete perturbations are encountered during walking, long-latency feedback responses to restore balance, presumably mediated by brainstem pathways (Macpherson and Fung, 1999; Deliagina et al., 2008; Honeycutt et al., 2009), are superimposed upon the locomotor pattern (Gorassini et al., 1994; Hiebert et al., 1994; Misiaszek, 2003; Bachmann et al., 2008). In anticipation of a perturbation or obstacle, modifications to the locomotor pattern are also observed (Tang et al., 1998; Bhatt et al., 2006) that are mediated by motor cortical activity (Drew et al., 2008).

Here, we tested the hypothesis that voluntary and reactive modifications to walking in humans could result from the recruitment of locomotor muscle synergies. Although muscle synergies have been examined in human walking (Clark et al., 2010) and balance control (Torres-Oviedo and Ting, 2007; Chvatal et al., 2011), it is not known whether common muscle synergies underlie these two behaviors. We demonstrate that a common set of locomotor muscle synergies can explain muscle activity in humans during overground walking at two walking speeds, reactive responses to discrete perturbations during walking, and anticipatory muscle activity before an expected perturbation. Our results 
corroborate the idea that muscle synergies can be flexibly recruited from a variety of hierarchical neural pathways for robust motor control, such as spinal pattern generators, brainstem pathways for balance, and descending cortical pathways.

\section{Materials and Methods}

We recorded muscle activity to ramp and hold translations of the support surface during overground walking in human subjects. Four perturbation directions were applied in the horizontal plane under the stance leg during two walking speeds: self-selected and slow, and the presentation order was randomized. We also examined walking conditions in which perturbations were not administered; these included trials in which the perturbations were either anticipated or not anticipated. Muscle synergies were extracted in $10 \mathrm{~ms}$ bins throughout the entire duration of the unperturbed walking trials. We then compared the muscle synergies used while walking at different speeds and under different anticipatory conditions. Finally, the muscle synergies from unperturbed walking were used to reconstruct the perturbed walking trials.

Data collection. Nine healthy subjects ( 4 male, 5 female) between the ages of 18 and 26 were exposed to support surface translations according to an experimental protocol that was approved by the Institutional Review Boards of Georgia Tech and Emory University. Subjects walked overground slowly $(0.6-0.7 \mathrm{~m} / \mathrm{s})$ or at a self-selected pace $(1.2-1.5 \mathrm{~m} / \mathrm{s})$ for $\sim 7.5 \mathrm{~m}$, or 7 gait cycles. The slow speed of $0.6 \mathrm{~m} / \mathrm{s}$ was selected because it is considered a slow walking speed adequate for independent living and is a clinically valid cutoff point of gait velocity (Studenski et al., 2003). Subjects listened to a metronome beat 4 times before they began walking each trial and were instructed to try to maintain that pace as closely as possible but the metronome was turned off while they were walking. Data collection began on the third step, to ensure the subject had reached a steady-state walking pattern and eliminate any variability associated with gait initiation. Eight trials of control walking at each speed were collected at the beginning of the experiment condition, in which the subject knew there would be no perturbation. In the perturbed walking conditions, subjects were told that there may or may not be a perturbation while they were walking. As subjects crossed a force plate halfway through the path, the platform translated in one of 4 equally spaced directions in the horizontal plane-anterior, posterior, medial, and lateral (displacement $12.4 \mathrm{~cm}$, velocity $40 \mathrm{~cm} / \mathrm{s}$, acceleration $0.7 \mathrm{~g}$ ). The perturbation was applied when the force under the right foot had reached $40 \%$ of body weight, occurring when the right leg was in early stance. Perturbation directions were randomized, and three trials of each direction for each speed were collected. For each speed, 12 trials of unperturbed walking were collected randomly in between perturbation trials to capture any anticipatory responses (unperturbed "catch" trials). The walking trials collected for each speed were blocked: a block of self-selected walking trials was collected and a block of slow walking trials was collected separately. The order of the conditions was randomized for each subject.

Since many muscles are required for muscle synergy analysis (Ting and Chvatal, 2010), surface electromyographic (EMG) activity was recorded from 16 lower back and leg muscles on the subject's right side, which was the stance leg in perturbed walking. The muscles recorded include: vastus lateralis (VLAT), rectus femoris (RFEM), rectus abdominis, biceps femoris, long head (BFLH), semitendinosus (SEMT), adductor magnus, erector spinae, abdominal external oblique, vastus medialis (VMED), tibialis anterior (TA), medial gastrocnemius (MGAS), lateral gastrocnemius (LGAS), soleus, peroneus (PERO), tensor fasciae latae (TFL), and gluteus medius. EMG data were sampled at $1080 \mathrm{~Hz}$, and then high-pass filtered at $35 \mathrm{~Hz}$, de-meaned, rectified, and low-pass filtered at $40 \mathrm{~Hz}$, using custom MATLAB routines. Additionally, kinetic data were collected from force plates under the feet (AMTI) and kinematic data were collected using a motion capture system (Vicon) and a custom 25-marker set that included head-arms-trunk, thigh, shank, and foot segments.

Data processing. EMG data were downsampled by averaging the data in $10 \mathrm{~ms}$ bins. At least three complete gait cycles for each trial were included in the analysis, which included the perturbed step cycle as well as at least one complete gait cycle both before and after the perturbed step cycle. Trials were concatenated end to end to form an $m \times n$ data matrix comprised of $m$ muscles by $n$ conditions (trials $\times$ time bins). In the unperturbed walking conditions, a matrix was formed consisting of $6-8$ walking trials with no perturbation. For visualization, each row of each data matrix (each muscle) was normalized to the maximum activation of the unperturbed self-selected speed walking trials. Therefore, the muscle activations ranged from 0 to 1 in unperturbed walking trials. Slow unperturbed walking trials as well as all perturbed walking trials were normalized by the same scaling factors.

Extraction of muscle synergies. We extracted muscle synergies from the data matrix of EMG recordings using non-negative matrix factorization, described by Lee and Seung (1999) and others (Tresch et al., 1999; Ting and Chvatal, 2010), which has previously been used for muscle synergy analysis (Ting and Macpherson, 2005; Torres-Oviedo and Ting, 2007). This is a linear decomposition technique that assumes that a muscle activation pattern, $\boldsymbol{M}$, in a given time period which was evoked by a perturbation in a particular direction is comprised of a linear combination of a few muscle synergies, $\mathbf{W}_{\mathbf{i}}$, that are each recruited by a synergy recruitment coefficient, $c_{i}$. Therefore, a particular muscle activation pattern at a particular time in response to a particular perturbation is represented by: $\boldsymbol{M}=c_{1} \mathbf{W}_{1}+c_{2} \mathbf{W}_{2}+c_{3} \mathbf{W}_{3}+\ldots$

$\mathbf{W}_{\mathbf{i}}$ is a vector corresponding to the $i$ th muscle synergy. Each component of $\mathbf{W}_{\mathbf{i}}$ represents the contribution of each muscle to that muscle synergy, and an individual muscle may contribute to multiple muscle synergies. Each $\mathbf{W}_{\mathbf{i}}$ is fixed across time, and each one is multiplied by a scalar recruitment coefficient, $c_{i}$, which changes over time and across conditions. The recruitment coefficient, $c_{i}$, is hypothesized to represent the neural command that specifies how that synergy is modulated over time, and how much each synergy will contribute to a muscle's total activity pattern (Ting, 2007).

Muscle synergy analysis. Muscle synergies were first identified from unperturbed walking patterns. Each row of the data matrix (each muscle) was normalized to have unit variance before extracting muscle synergies 


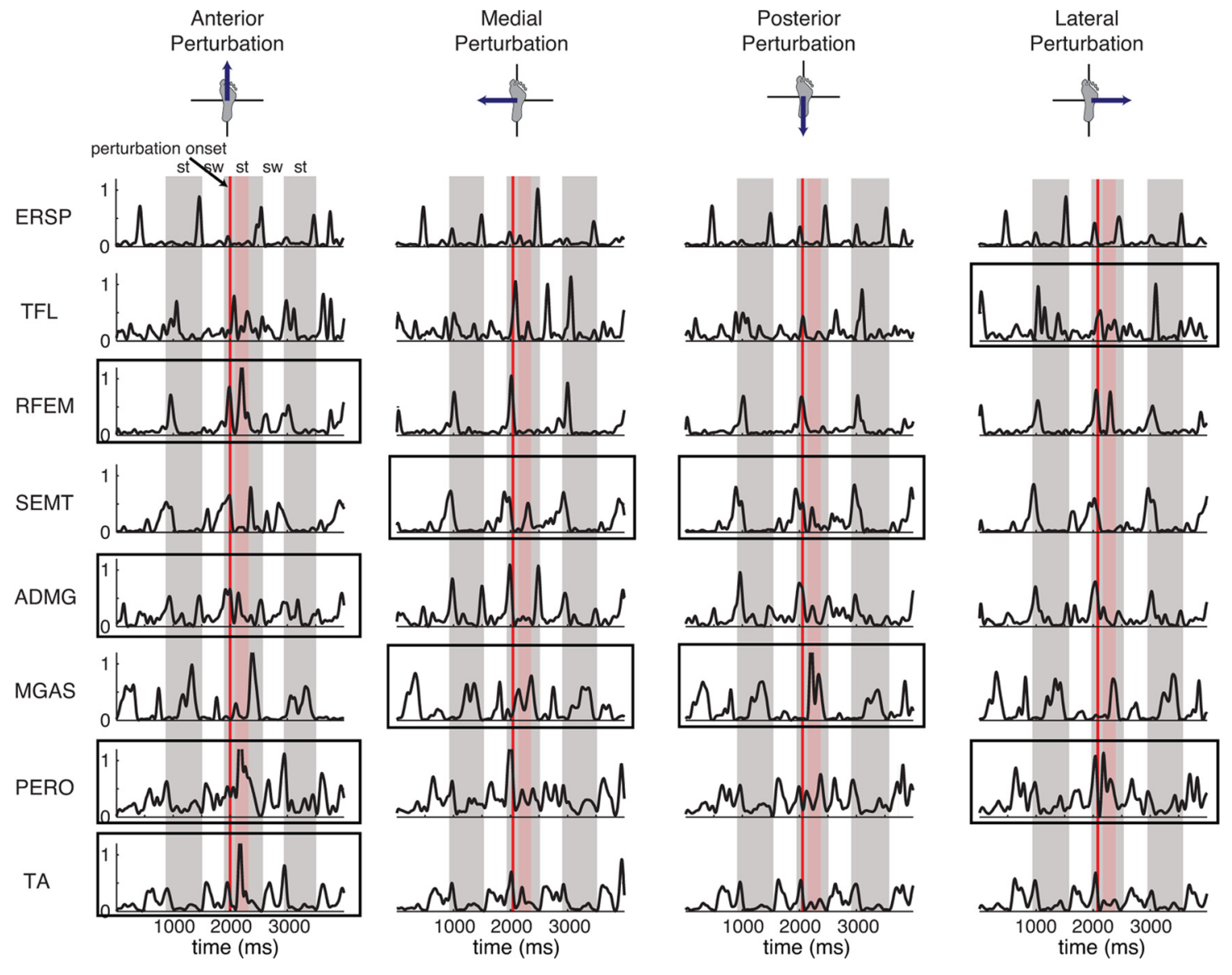

Figure 2. EMGs during perturbed self-selected speed walking across perturbation directions. Shown are example trials for each perturbation direction in one subject. Red vertical lines indicate perturbation onset. Gray shaded boxes indicate stance phase. The red shaded box indicates time window $100-400 \mathrm{~ms}$ after perturbation that contains the expected postural response resulting from the perturbation. Different muscles were activated during the perturbation response depending on the perturbation direction (indicated by boxes).

Table 1. Number of muscles activated differently in postural responses during walking compared with unperturbed walking, and swing leg (left leg) step length and width for walking without perturbations and for each direction of perturbation at each walking speed

\begin{tabular}{|c|c|c|c|c|c|c|c|c|}
\hline \multirow[b]{2}{*}{$\begin{array}{l}\text { Perturbation } \\
\text { direction }\end{array}$} & \multicolumn{4}{|c|}{ Self-selected walking } & \multicolumn{4}{|l|}{ Slow walking } \\
\hline & $\begin{array}{l}\text { Number of EMG } \\
\text { changes }\end{array}$ & $\begin{array}{l}\text { Right stance } \\
\text { duration (ms) }\end{array}$ & $\begin{array}{l}\text { Left step } \\
\text { length (mm) }\end{array}$ & $\begin{array}{l}\text { Left step } \\
\text { width (mm) }\end{array}$ & $\begin{array}{l}\text { Number of } \\
\text { EMG changes }\end{array}$ & $\begin{array}{l}\text { Right stance } \\
\text { duration (ms) }\end{array}$ & $\begin{array}{l}\text { Left step } \\
\text { length (mm) }\end{array}$ & $\begin{array}{l}\text { Left step } \\
\text { width (mm) }\end{array}$ \\
\hline None & $\mathrm{N} / \mathrm{A}$ & $565 \pm 12$ & $592 \pm 15$ & $-117 \pm 32$ & N/A & $1181 \pm 170$ & $544 \pm 23$ & $-112 \pm 37$ \\
\hline Anterior & $7.6 \pm 2.2$ & $644 \pm 13$ & $511 \pm 48$ & $-166 \pm 56$ & $9.2 \pm 2.5$ & $1272 \pm 179$ & $468 \pm 10$ & $-117 \pm 41$ \\
\hline Medial & $7.9 \pm 2.9$ & $569 \pm 25$ & $571 \pm 56$ & $59 \pm 29$ & $8.4 \pm 4.2$ & $1219 \pm 209$ & $510 \pm 32$ & $156 \pm 41$ \\
\hline Posterior & $6.4 \pm 3.4$ & $497 \pm 10$ & $623 \pm 57$ & $-133 \pm 35$ & $4.5 \pm 3.0$ & $883 \pm 66$ & $611 \pm 16$ & $-126 \pm 8$ \\
\hline Lateral & $7.9 \pm 3.4$ & $533 \pm 8$ & $492 \pm 28$ & $-271 \pm 17$ & $5.9 \pm 2.2$ & $825 \pm 43$ & $376 \pm 35$ & $-298 \pm 28$ \\
\hline
\end{tabular}

Magnitude of EMG activation during the window $100-400 \mathrm{~ms}$ following perturbation onset was calculated and expressed as a ratio relative to the average EMG activation during a comparable window in unperturbed walking. Significan differences were determined using ANOVA followed by Dunnett's test $(\alpha=0.05)$, and the number of muscles different from unperturbed walking are tallied here. Step lengths and widths are relative to the right foot. Also displayed are right leg stance times for the stance phase that includes the perturbation. Shown are the mean and SD for each perturbation direction across all subjects.

to ensure each muscle was equally weighted in the extraction; this normalization was removed after muscle synergy extraction, returning the data and muscle synergies back to the original scaling. To determine whether bin size affected the muscle synergy structure, and to validate that the identified muscle synergies underlying walking were robust across bin sizes, we also extracted muscle synergies from data binned in $25,50,100$, and $200 \mathrm{~ms}$ bins.

To determine whether anticipation of a perturbation affected the walking pattern and which muscle synergies were recruited during this anticipation, muscle synergies were extracted separately from unperturbed walking trials in which the subjects knew there would be no perturbation, and from unperturbed walking "catch" trials. Furthermore, to determine whether walking speed affected muscle synergy recruitment, muscle synergies were extracted separately from self-selected walking catch trials and slow walking catch trials. Similarity between muscle syn- ergies extracted from each speed individually was quantified by calculating the correlation coefficient $(r)$ between the muscle synergy vectors. A pair of muscle synergies having $r>0.623$ were considered similar, which corresponds to the critical value of $r^{2}$ for 16 muscles $\left(r^{2}=0.388\right.$; $p=$ 0.01 ). Additionally, we generated a distribution of $Z$ scores for similarity of muscle synergies across conditions based on chance by comparing the muscle synergies from one condition (e.g., self-selected walking) with 22,000 random permutations of the elements of muscle synergies from another condition (e.g., slow walking), and transforming these $r$ values into $Z$ scores. An $r$ of $>0.623$ corresponds to a $Z$ score of $>2.409$, indicating a pair of muscle synergies is statistically more similar than would be expected by chance ( $p<0.008$; see Chvatal et al., 2011, for muscle synergy comparison details). Because similar muscle synergies were recruited during walking at different speeds, and when perturbations were expected or not, one set of muscle synergies was then extracted from a 
A Muscle synergies from different walking speeds

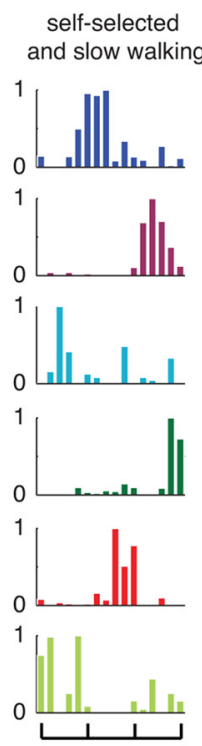

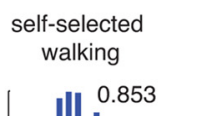

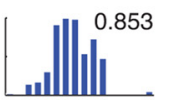

[...

0.969
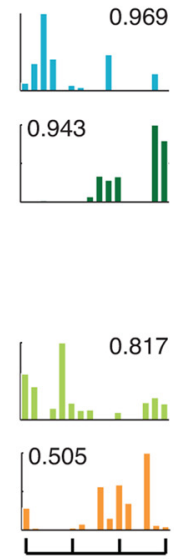

slow walking
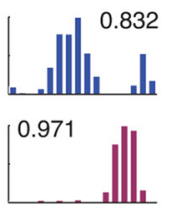

0.819
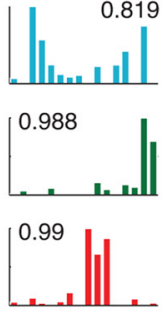

0.906

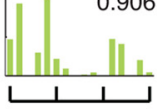

B Effect of anticipation on synergies
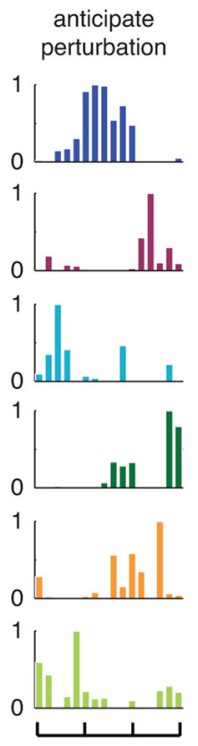

no anticipation
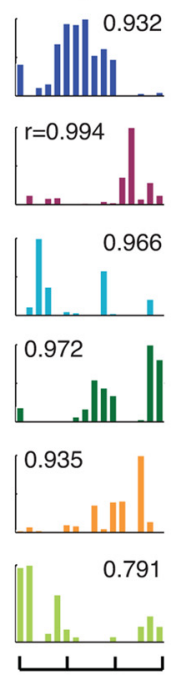

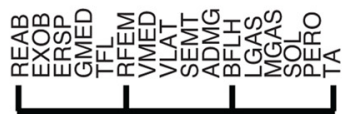

Figure 3. Robustness of muscle synergies across unperturbed walking conditions. $A$, Muscle synergies extracted from different walking speeds were similar. Shown are example muscle synergies from one subject extracted from slow walking trials and self-selected speed walking trials combined, slow walking catch trials, and self-selected speed walking catch trials. Correlations between each muscle synergy vector and the corresponding muscle synergy from combined self-selected and slow walking data are shown. $\boldsymbol{B}$, Muscle synergies extracted from unperturbed self-selected speed walking with and without anticipation have similar muscle synergy composition. Shown are muscle synergies extracted from walking catch trials (with anticipation) and walking control trials (no anticipation). Correlations between muscle synergy vectors are shown.

matrix consisting of both self-selected walking catch trials and slow walking catch trials, and these muscle synergies were termed "locomotor" muscle synergies. We extracted muscle synergies from catch trials because they reflected the ongoing walking pattern at the time of perturbation.

The number of muscle synergies required to explain any of these datasets was determined by selecting the smallest number of synergies that could adequately reconstruct the muscle responses during the unperturbed walking condition (Nsyn). The goodness of fit of the data reconstruction using the muscle synergies was quantified by variability accounted for (VAF), defined as $100 \times$ uncentered coefficient of determination (Zar, 1999; Torres-Oviedo et al., 2006). Nsyn accounted for $>85 \%$ VAF overall. We added the further local criterion that muscle synergies also account for $>75 \%$ VAF in each muscle. This local fit criterion was more stringent and ensured that relevant features of the dataset are reproduced. The number of muscle synergies was increased if local fits were improved. However, if an additional muscle synergy contributed evenly to the VAF across muscles and perturbation directions, it was not included because it likely represented noise in the data rather than variations due to trial or perturbation direction. Nsyn was also validated using factor analysis: 1-12 factors were extracted and the $\log$ likelihood of each was plotted versus the number of factors. Nsyn was chosen by finding the point on the log-likelihood curve where curvature was greatest (Tresch et al., 2006).

Data analysis. To quantify kinematic modifications in the perturbed gait cycle, we calculated left leg step length and step width, as well as right leg stance time. Left leg step length and width were found by subtracting the heel marker position of the right foot from the heel marker position of the left foot at the beginning of left stance immediately following the perturbation. The anterior/posterior distance between the left heel and right heel was step length and the medial/lateral distance between the left heel and right heel was step width. These were averaged across trials of the same perturbation direction for each speed and compared with the left step length and width in unperturbed walking catch trials for the step in which the subject crossed the force plate. Additionally, right foot stance times were averaged across perturbed walking trials of the same direction for each speed and compared with unperturbed walking stance times. Stance phase for each foot was determined using heel marker positions.

To investigate whether locomotor muscle synergies of the ongoing walking pattern were also recruited during perturbations to walking, locomotor muscle synergies were used to reconstruct the perturbed walking trials. VAF and $r^{2}$ correlations between measured electromyograms in $10 \mathrm{~ms}$ bins and reconstructed EMGs were computed for all trials for the entire trial duration as well as for a time window $100-400 \mathrm{~ms}$ after perturbation onset to determine how well the locomotor muscle synergies could explain the perturbation response. As we did not observe differences in the quality of reconstruction at different latencies following perturbations, we used the $100-400 \mathrm{~ms}$ time window (e.g., thirty 10 ms bins) to compute summary statistics describing the goodness of fit $\left(r^{2}\right.$ and VAF) of the reconstruction of the relatively short perturbation response only. This was because the $r^{2}$ and VAF for the entire trial duration was dominated by the fit to the ongoing locomotor pattern. VAF provides a measure of how well the magnitude of the measured EMG and reconstructed EMG match, whereas $r^{2}$ quantifies how well the shapes of the curves match.

To identify which muscle synergies (or muscles) were recruited during postural responses in walking, we quantified the additional walking muscle synergy recruitment required during perturbation responses when compared with unperturbed walking. To this end, we found the average recruitment coefficient $\left(C_{\text {mag }}\right.$ ) for each muscle synergy during the time window $100-400 \mathrm{~ms}$ following perturbation onset for each perturbation trial. Likewise, we found the average recruitment coefficient $\left(C_{\text {mag }}\right)$ for the same time window in unperturbed walking catch trials, although instead of based on perturbation onset, this window was based on the vertical force under the right foot corresponding to the force under the foot when a perturbation was triggered in perturbation trials. We averaged $C_{\text {mag }}$ for each muscle synergy across perturbation trials with perturbations in the same direction, and expressed these as percentages of the unperturbed walking $C_{\text {mag }}$, for each walking speed. A one-way ANOVA 
Self-selected Walking

Synergy Coefficients (C)
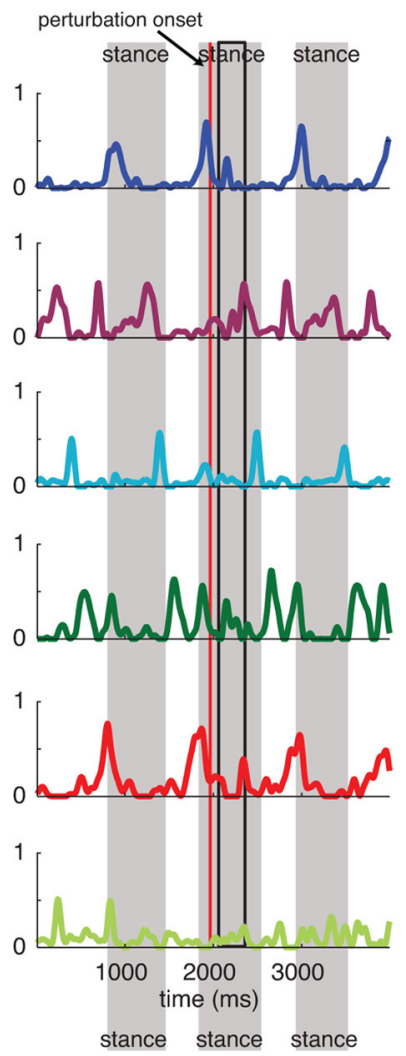

Muscle Synergies (W)
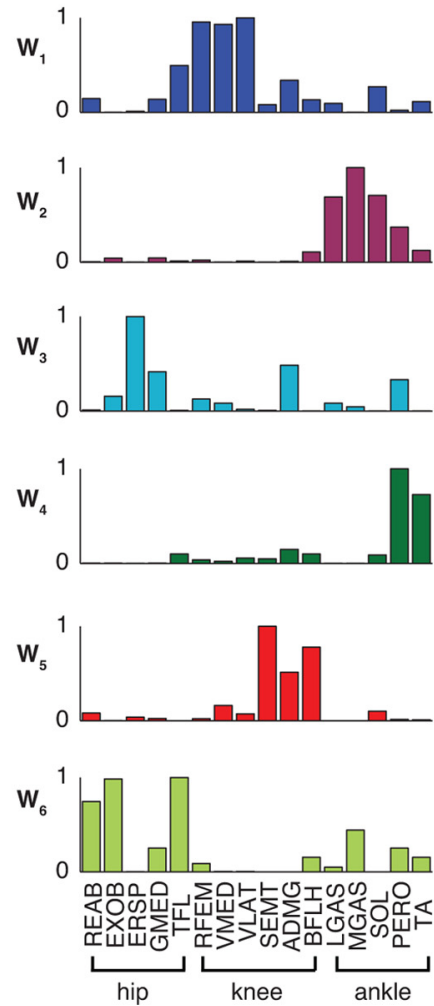

Slow Walking

Synergy Coefficients (C)

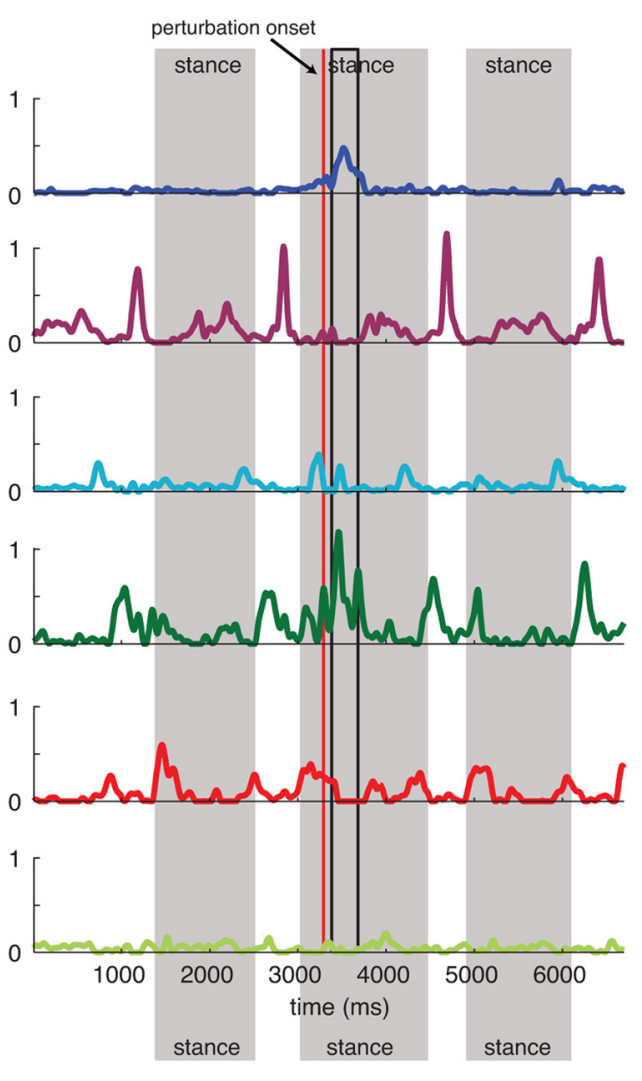

Figure 4. Example of locomotor muscle synergies and recruitment coefficients during self-selected and slow walking trials containing anterior perturbations. Each muscle synergy (W) defines a spatial pattern of muscle activity across multiple muscles, some of which span multiple joints and may have different biomechanical functions (Table 2). Each muscle synergy is temporally recruited (C) during a particular phase of the gait cycle (Table 2), but the amplitude of recruitment may vary from step to step. In slow walking, recruitment of some muscle synergies is greatly reduced or absent. Following an anterior perturbation, muscle synergies are additionally recruited at $\sim 100 \mathrm{~ms}$ latency following perturbation onset (red vertical line) as part of the feedback response to perturbation (black box). This results in some muscle synergies being active in mid-stance on the perturbed step whereas they may be typically recruited in other gait phases.

Table 2. Description of locomotor muscle synergies and comparison to previous work (Ting et al., 1999; Ivanenko et al., 2004; Clark et al., 2010)

\begin{tabular}{|c|c|c|c|c|c|c|}
\hline & Function & Timing & Major muscle contributions & Clark & Ivanenko & Ting \\
\hline$W_{1}$ & Body support and propulsion & Early stance & RFEM, VMED, VLAT & $\mathrm{C} 1$ & Factor 2 & Extension \\
\hline$W_{2}$ & Plantar flexion/body support and propulsion & Mid-late stance & MGAS, LGAS, SOL & $\mathrm{C} 2$ & Factor 1 & Plantar flexion \\
\hline$W_{3}$ & Trunk extension/adduction & Late stance & ERSP, GMED, ADMG & & Factor 4 & \\
\hline$W_{4}$ & Ankle dorsiflexion/eversion, leg deceleration & Early swing and swing-to-stance transition & PERO, TA & C3 & Factor 5 & Dorsiflexion \\
\hline$W_{5}$ & Leg deceleration & Late swing/early stance & SEMT, ADMG, BFLH & C4 & Factor 3 & Posture \\
\hline$W_{6}$ & Trunk flexion/abduction & Early stance and throughout gait cycle & REAB, EXOB, TFL & & & \\
\hline
\end{tabular}

ADMG, Adductor magnus; ERSP, erector spinae; EXOB, abdominal external oblique; GMED, gluteus medius; REAB, rectus abdominis; SOL, soleus.

(perturbation direction) followed by Dunnett's test for comparing group means to a control mean (Zar, 1999) was performed for each muscle synergy of each subject to compare muscle synergy recruitment during perturbation responses to unperturbed walking trials. The same procedure was used to quantify differences in individual muscle activity during responses to perturbations compared with unperturbed walking.

Similarly, to identify which muscle synergies were recruited in anticipation of a perturbation we quantified the additional walking muscle synergy recruitment just before perturbation onset. We averaged the magnitude of the recruitment coefficient curve $\left(C_{\text {mag }}\right)$ for each muscle synergy for the $100 \mathrm{~ms}$ before perturbation onset during self-selected speed walking, and for $200 \mathrm{~ms}$ before perturbation onset during slow walking. These two time windows correspond to $15 \%$ of the average duration of stance for each walking speed. Likewise, we found the average magnitude of the recruitment coefficient curve $\left(C_{\text {mag }}\right)$ for each muscle synergy for the same time windows in walking trials in which the subject knew there would be no perturbation, and therefore no anticipation (again these windows were based on the vertical force under the right foot which corresponded to the force under the foot when a perturbation was triggered in perturbation trials). We averaged $C_{\text {mag }}$ for each muscle synergy across perturbation and catch trials, and expressed these as percentages of the unperturbed walking $C_{\text {mag }}$, for each walking speed. A t test was performed for each subject to compare each muscle synergy recruitment coefficient in anticipation of a perturbation to the recruitment coefficient used during control walking trials, using $p<0.05$ to determine significant differences.

To determine whether the same locomotor muscle synergies were consistently used across subjects, we grouped muscle synergies and calculated the correlation coefficients $(r)$ between all synergy vectors across all subjects. As above, similar muscle synergies were defined as those having $r>0.623$, which corresponds to the critical value of $r^{2}$ for 16 muscles $\left(r^{2}=0.388 ; p=0.01\right.$; see Chvatal et al., 2011 for muscle synergy comparison details). To determine whether there were any additional similar muscle synergies across subjects while allowing for individual differences 


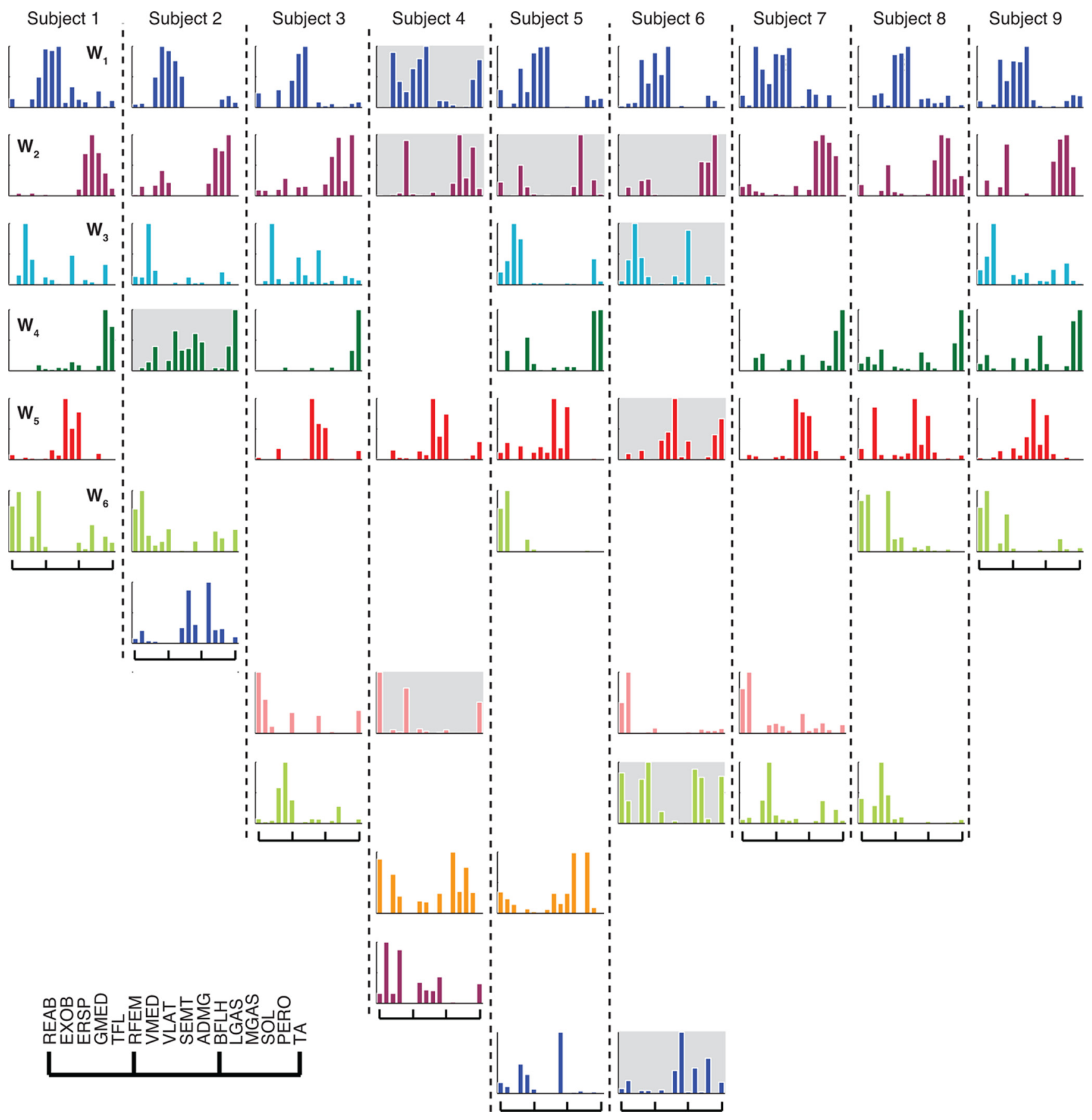

Figure 5. Comparison of locomotor muscle synergies across all subjects. Similar muscle synergies have $r>0.623(p=0.01)$ when compared with the corresponding muscle synergy found in Subject 1. Synergies indicated with a gray background have $r>0.497(p=0.05)$, but $<0.623$ when compared with Subject 1 . Two muscle synergies were similar across all subjects $\left(\mathbf{W}_{1}\right.$ and $\left.\mathbf{W}_{2}\right)$, and another was similar across eight subjects $\left(\mathbf{W}_{\mathbf{5}}\right)$. One muscle synergy was similar across 7 subjects $\left(\mathbf{W}_{\mathbf{4}}\right)$, one was similar across 6 subjects $\left(\mathbf{W}_{\mathbf{3}}\right)$, and one was similar across 5 subjects $\left(\mathbf{W}_{\mathbf{6}}\right)$.

when comparing across subjects, we also noted those muscle synergy pairs having $r>0.497$, which corresponds to the critical value of $r^{2}$ for 16 muscles using $p=0.05$.

\section{Results}

For each subject, muscle synergies extracted from unperturbed walking were sufficient to reproduce variations in the muscle activation patterns due to different walking speeds, anticipation before an expected perturbation, reactive feedback responses to multidirectional perturbations encountered while walking, as well as altered step mechanics following a perturbation. Our locomotor muscle synergies were similar to those found in prior studies, with the addition of muscle synergies associated with medial and lateral limb control.

\section{Differences in EMG activity and gait kinematics during unperturbed and perturbed walking}

The basic timing of muscle activity associated with the rhythmic locomotor pattern for walking was similar across all trials. As previously described (Winter and Yack, 1987), we found that in both self-selected and slow walking speeds, TA and PERO were active during swing and in early stance, quadriceps (VLAT, VMED, and RFEM) were active at the end of swing and in early 
A Recruitment of muscle synergies during anticipation of a perturbation (slow walking)

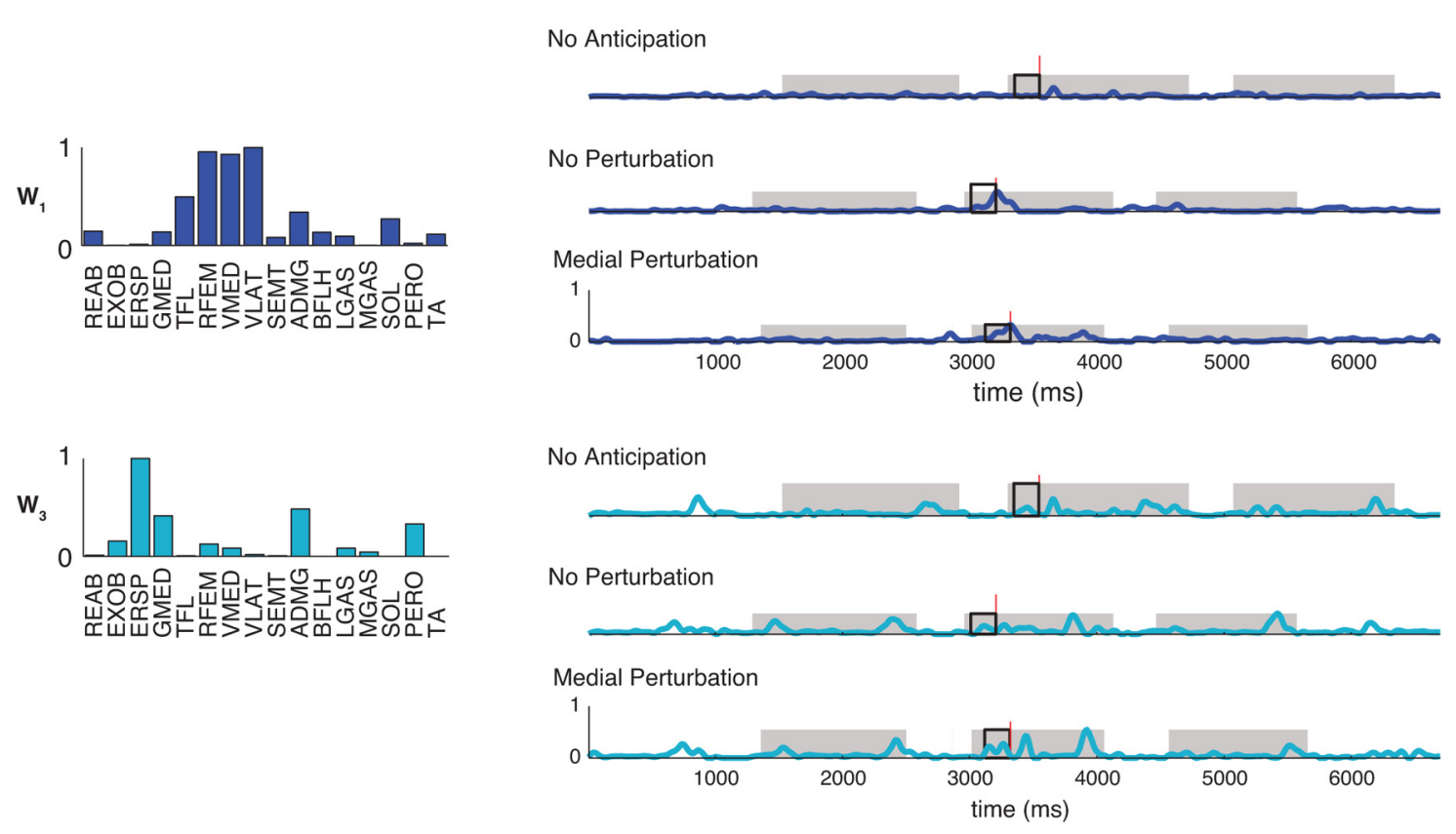

B Muscle synergy recruitment evoked by anticipation

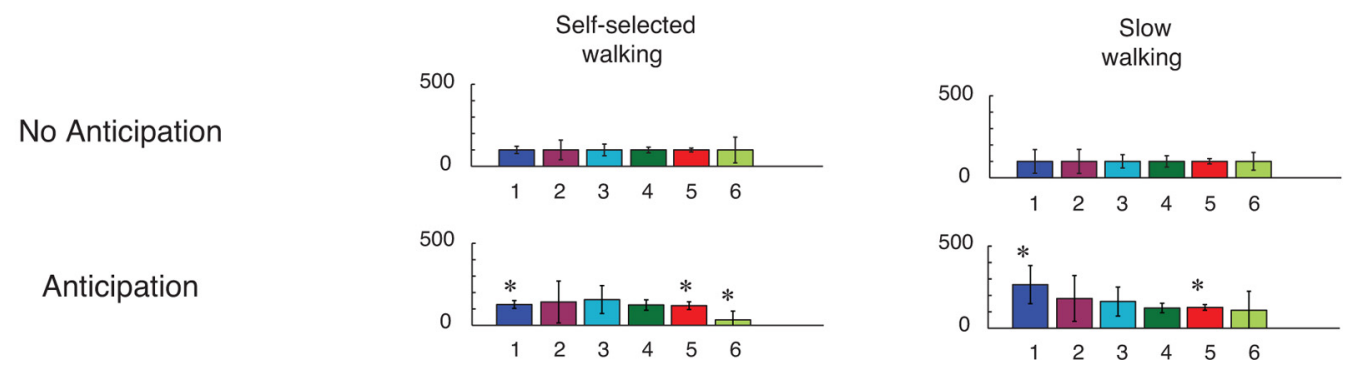

Figure 6. Changes in muscle synergy recruitment due to anticipation of a perturbation during walking. $A$, Example muscle synergy recruitments are shown during slow walking for a control walking trial in which a perturbation was not expected (no anticipation), a walking catch trial in which a perturbation was expected but not delivered (no perturbation), and a perturbed walking trial. For both muscle synergies $\left(\mathbf{W}_{\mathbf{1}}\right.$ and $\mathbf{W}_{3}$ ), muscle synergy recruitment was observed during a window of time (black box) before perturbation onset (red line) when a perturbation was anticipated. $\boldsymbol{B}$, Average and SD of the percentage of muscle synergy recruitment during the time window preceding perturbations in anticipation trials compared with no trials with no anticipation. ${ }^{*} p<0.05$, significant differences using $t$ tests. Generally $2-3$ muscle synergies were recruited to a greater extent during the anticipation window for each walking speed.

stance, hamstrings (SEMT, BFLH) were active during early stance, and gastrocnemii muscles (LGAS, MGAS) were active during midstance. In slow walking, additional activity was found in hamstrings (SEMT, BFLH) in late stance, and gastrocnemii during swing. These basic locomotor patterns were also preserved for step cycles before and after the perturbed step cycle (Figs. 1, 2).

However, the observed magnitude of muscle activity and perturbation responses depended on both walking speed and perturbation direction. Locomotor EMGs in several muscles were higher at self-selected versus slow walking speeds (Fig. 1). Further, feedback responses to identical perturbations were larger in slow walking relative to the ongoing walking pattern, compared with self-selected walking responses. (Fig. 1, red shaded area). Feedback responses also recruited different muscles (Fig. 2, boxes) and different numbers of muscles (Table 1) depending upon perturbation direction. As expected based on standing balance control (Henry et al., 1998; TorresOviedo and Ting, 2007), anterior perturbations elicited responses in TA, PERO, and quadriceps, whereas posterior and medial perturbations elicited postural responses in SEMT and MGAS. Lateral perturbations elicited posture responses in TFL and PERO (Fig. 2).

Following a perturbation, locomotor mechanics were altered compared with control walking, as indicated by the differential placement of the swing leg following the perturbation (Table 1). The right leg stance duration was increased in anterior perturbations, consistent with previous work (Tang et al., 1998), likely due to the additional time required to restore the center of mass motion to a forward direction after the perturbation caused it to accelerate backward. Right leg stance duration was decreased in posterior perturbations as subjects quickly stepped the left foot down to continue walking forward. Longer steps in the left (swing) leg were observed following posterior perturbations, and shorter steps followed anterior perturbations. Lateral perturbations resulted in wider steps, consistent with previous work (Oddsson et al., 2004), whereas medial perturbations generally resulted in cross-over steps similar to perturbation responses in standing balance (Maki et al., 1996). 
A Recruitment of muscle synergies during unperturbed and perturbed walking
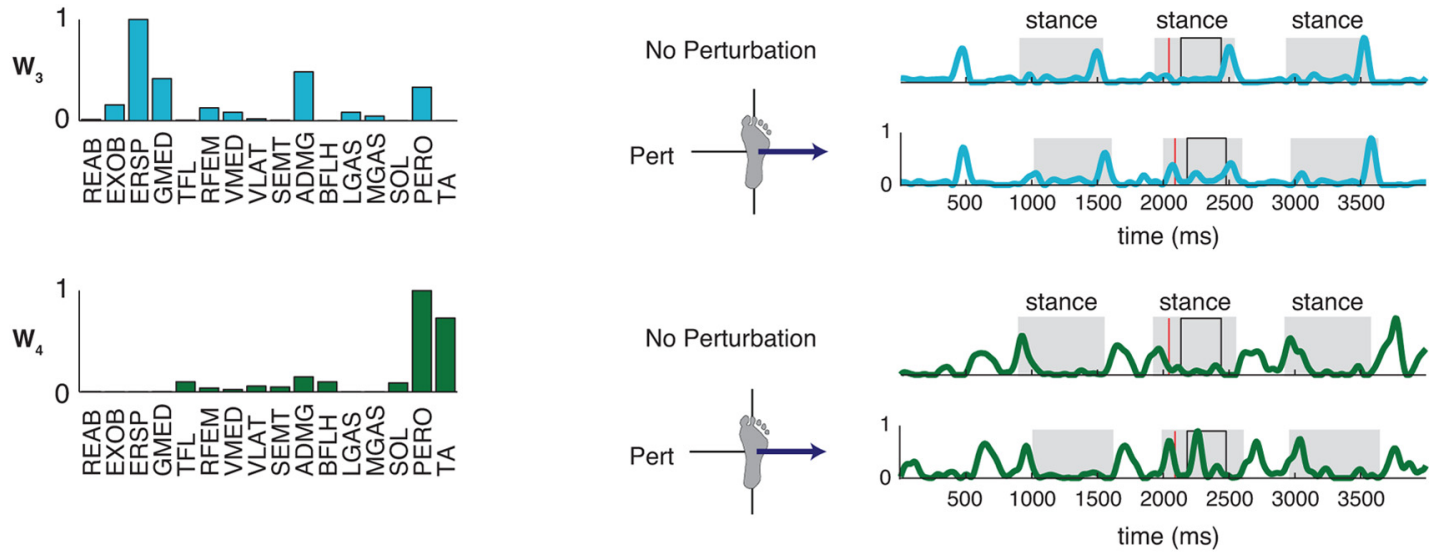

\section{B Muscle synergy recruitment evoked by perturbation}

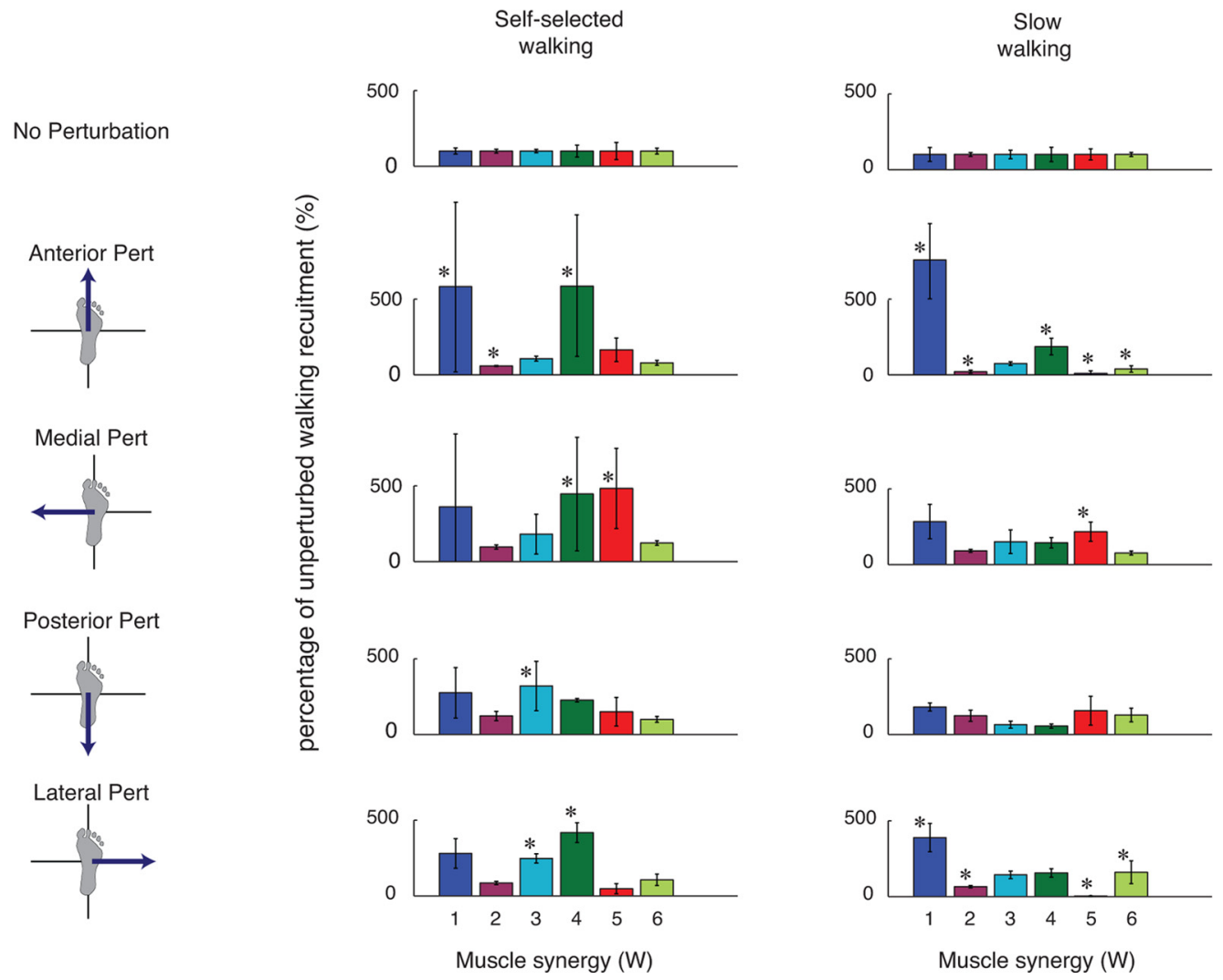

Figure 7. Changes in muscle synergy recruitment due to perturbation responses during walking. $\boldsymbol{A}$, Example muscle synergy recruitments are shown during self-selected walking for a trial of unperturbed walking and a lateral perturbation trial. Both muscle synergies $\left(\mathbf{W}_{\mathbf{3}}\right.$ and $\left.\mathbf{W}_{\mathbf{4}}\right)$ shown are additionally recruited during the $100-400$ ms latency time bin (black box) following perturbation onset (red lines). $\boldsymbol{B}$, Average and SD of the magnitudes of muscle synergy recruitment in perturbation responses during walking expressed as a percentage of unperturbed walking recruitment magnitudes during the 100 - 400 ms time window following perturbation onset. Different patterns of muscle synergy recruitment were observed across perturbation directions and walking speeds. ${ }^{*} \alpha=0.05$, significant differences using ANOVA followed by Dunnett's test. Generally $2-4$ muscle synergies were recruited to a greater or lesser extent during the perturbation response for each perturbation direction. Different muscle synergies were recruited during perturbation responses in different directions.

\section{Consistent spatial structure of muscle synergies across} walking conditions

Six to eight muscle synergies extracted from $10 \mathrm{~ms}$ time bins were sufficient to explain $89 \pm 3 \%$ of the overall variability in selfselected and slow unperturbed walking trials across subjects, and $89 \pm 6 \%$ of the variability observed in each muscle. For all further analysis we extracted muscle synergies from 10 ms time bins, which were nearly identical to those extracted from all time points ( $r=0.98 \pm 0.02)$. However, we verified that muscle synergy composition was similar when muscle synergies were extracted from all time points compared with bin sizes of $10 \mathrm{~ms}, 25$ $\mathrm{ms}$, and $50 \mathrm{~ms}(r=0.97 \pm 0.03$, data not shown). For bin sizes of 100 or $200 \mathrm{~ms}$, one muscle synergy differed from those extracted from all time points $(r=0.40)$, although the remaining muscle 
synergies were similar to those extracted from all time points $(r=$ $0.85 \pm 0.08)$.

Consistent with prior studies (Hof et al., 2002; Clark et al., 2010), we found muscle synergies within each subject to be robust across walking speeds. Muscle synergies extracted individually from either self-selected or slow walking speeds were similar to those extracted from the combined dataset $(r=0.90 \pm 0.07$, Fig. $3 A$ ) across all subjects. In six subjects we identified one muscle synergy that was only used at one walking speed, usually composed of hamstring or trunk muscles. These muscle synergies were also identified using the combined self-selected and slow walking data in four of these six subjects (Fig. 3A).

Muscle synergy composition was generally unaffected by anticipation of a perturbation. In six of nine subjects, similar muscle synergies were identified in walking trials when a perturbation was not expected versus walking catch trials, where no perturbation was given but one was expected $(r=0.93 \pm 0.07$, Fig. $3 B)$. In two subjects, an additional muscle synergy comprised of either the hamstring and/or trunk muscles was identified in walking catch trials but not in control walking trials. In one subject, an additional muscle synergy with strong contribution from TFL was identified in control walking trials but not walking catch trials.

\section{Muscle synergies extracted from unperturbed walking}

Locomotor muscle synergies were similar to those reported previously (Neptune et al., 2009; Clark et al., 2010), with the addition of muscle synergies involving trunk muscles, adductors and abductors reflecting 16 versus 8 EMGs recorded. In control walking trials and unperturbed step cycles of perturbed walking trials, muscle synergies were generally recruited during particular phases of the gait cycle (Fig. 4, Table 2). However, a few muscle synergies were recruited throughout the gait cycle, composed usually of trunk muscles, such as $\mathbf{W}_{\mathbf{6}}$. We found that muscle synergies could be approximately characterized by the same functional descriptors as in prior studies based on their muscle composition as well as on timing of recruitment during the locomotor cycle (Table 2). Although our methodology differs from that of Ivanenko et al. (2004), the cyclic nature of walking suggests that fixed temporal features they found can be compared with the fixed spatial features identified in ours and other studies in the case of unperturbed walking, because muscle groups are generally activated at consistent phases in the gait cycle. However, reactive feedback responses cannot be described using fixed temporal features of locomotion, as they depend upon the timing and characteristics of the perturbation (Welch and Ting, 2009), and are better described by fixed spatial rather than temporal features (Safavynia and Ting, 2012).

As previously found in postural responses (Torres-Oviedo and Ting, 2007; Clark et al., 2010), muscle synergy number (6-8) and structure was specific to each subject, but commonalities were evident in muscle synergy composition and recruitment across subjects (Fig. 5). Generally, the muscle synergies used by all or most of the subjects are similar to those reported previously (Clark et al., 2010). $\mathbf{W}_{\mathbf{1}}$ and $\mathbf{W}_{\mathbf{2}}$ were similar across all nine subjects ( $r=0.78 \pm 0.12$, and $r=0.73 \pm 0.14$, respectively). $\mathbf{W}_{4}$ was similar across 7 subjects $(r=0.77 \pm 0.14)$ and $\mathbf{W}_{5}$ was similar across 8 subjects $(r=0.86 \pm 0.15)$. The largest individual differences were observed in muscle synergies used for trunk stabilization: $\mathbf{W}_{\mathbf{3}}$ was similar across 6 subjects $(r=0.73 \pm 0.13)$, and $\mathbf{W}_{\mathbf{6}}$ was similar across 5 subjects $(r=0.82 \pm 0.12)$. A few other muscle synergies were unique to individual subjects (Fig. 5).
Table 3. Number of muscle synergies recruited differently in postural responses during walking compared with unperturbed walking

\begin{tabular}{lll}
\hline & \multicolumn{2}{l}{$\begin{array}{l}\text { Number of muscle synergies recruited differently } \\
\text { from unperturbed walking }\end{array}$} \\
\cline { 2 - 3 } Perturbation direction & Self-selected walking & Slow walking \\
\hline Anterior & $3.8 \pm 1.1$ & $4.2 \pm 1.1$ \\
Medial & $2.9 \pm 1.4$ & $3.2 \pm 1.8$ \\
Posterior & $2.0 \pm 1.3$ & $1.7 \pm 1.2$ \\
Lateral & $3.0 \pm 1.2$ & $2.9 \pm 1.8$ \\
\hline
\end{tabular}

Magnitude of muscle synergy recruitment during the window $100-400$ ms following perturbation onset was calculated and expressed as a ratio relative to the average muscle synergy recruitment during a comparable window in unperturbed walking catch trials. Significant differences were determined using ANOVA followed by Dunnett's test $(\alpha=0.05$ ), and the number of muscle synergies different from unperturbed walking are tallied here. Shown are the mean and SD for each perturbation direction across all subjects.

\section{Varying temporal recruitment of locomotor muscle synergies in perturbed walking}

Anticipatory muscle activity before stepping on the force plate could be attributed to changes in the temporal recruitment of muscle synergies used in walking (Fig. 6). For example, $\mathbf{W}_{\mathbf{1}}$ was recruited in slow walking during the time window $200 \mathrm{~ms}$ before perturbation onset during trials in which a perturbation was expected, regardless of whether a perturbation was delivered (Fig. $6 A)$. In self-selected speed walking, $\mathbf{W}_{1}$ and $\mathbf{W}_{5}$ were recruited during the time window immediately preceding the perturbation onset to a greater extent than they were recruited during a corresponding time window in walking trials in which subjects knew they would not be perturbed, and $\mathbf{W}_{6}$ recruitment was suppressed (Fig. $6 \mathrm{~B}$ ). In slow walking, $\mathbf{W}_{\mathbf{1}}$ and $\mathbf{W}_{5}$ were recruited in anticipation of a perturbation (see also Fig. 4, right). Across subjects, $2.8 \pm 1.3$ muscle synergies were recruited differently in anticipation of a perturbation than they were recruited when a perturbation was not expected in self-selected walking, and $1.1 \pm$ 1.1 muscle synergies were recruited differently in slow walking.

Reactive responses to perturbations differentially recruited locomotor muscle synergies depending on perturbation direction (Fig. 7). Consistent with postural responses during standing balance (Horak and Macpherson, 1996; Torres-Oviedo and Ting, 2010), reactive responses to perturbations during walking occurred between 100 and $400 \mathrm{~ms}$ after the onset of the perturbation. In self-selected walking, although $\mathbf{W}_{1}$ and $\mathbf{W}_{4}$ were active following perturbations in all directions providing propulsion to continue walking forward, the largest responses were observed following anterior perturbations, presumably to counteract the body falling backward (Figs. 4, 7B). A similar pattern was seen in slow walking, but only for $\mathbf{W}_{1}$. Large responses were also observed following medial perturbations where $\mathbf{W}_{\mathbf{5}}$ (hamstrings) was recruited in addition to $\mathbf{W}_{\mathbf{1}}$ and $\mathbf{W}_{\mathbf{4}}$ (Fig. $7 B$ ). In lateral perturbations $\mathbf{W}_{4}$ was recruited strongly to counteract induced ankle inversion and in posterior perturbations $\mathbf{W}_{\mathbf{3}}$ was recruited to return the trunk upright (Fig. $7 B$ ). On average across subjects, 3-4 muscle synergies were recruited differently compared with unperturbed walking in anterior perturbations (Table 3), which cause the body to move backward, opposing the task goal of walking forward, whereas only 1-2 muscle synergies were recruited differently from unperturbed walking following posterior perturbations that cause the body to move forward, consistent with the task goal of moving the body forward (Table 3 ).

We were able to predict all muscle activity from both walking speeds and all perturbation directions by time-varying recruitment of locomotor muscle synergies $\left(\mathrm{VAF}=87.8 \pm 8.2 \%, r^{2}=\right.$ $0.75 \pm 0.20)$. We found that this was still true when the time window was reduced to specifically examine the postural re- 

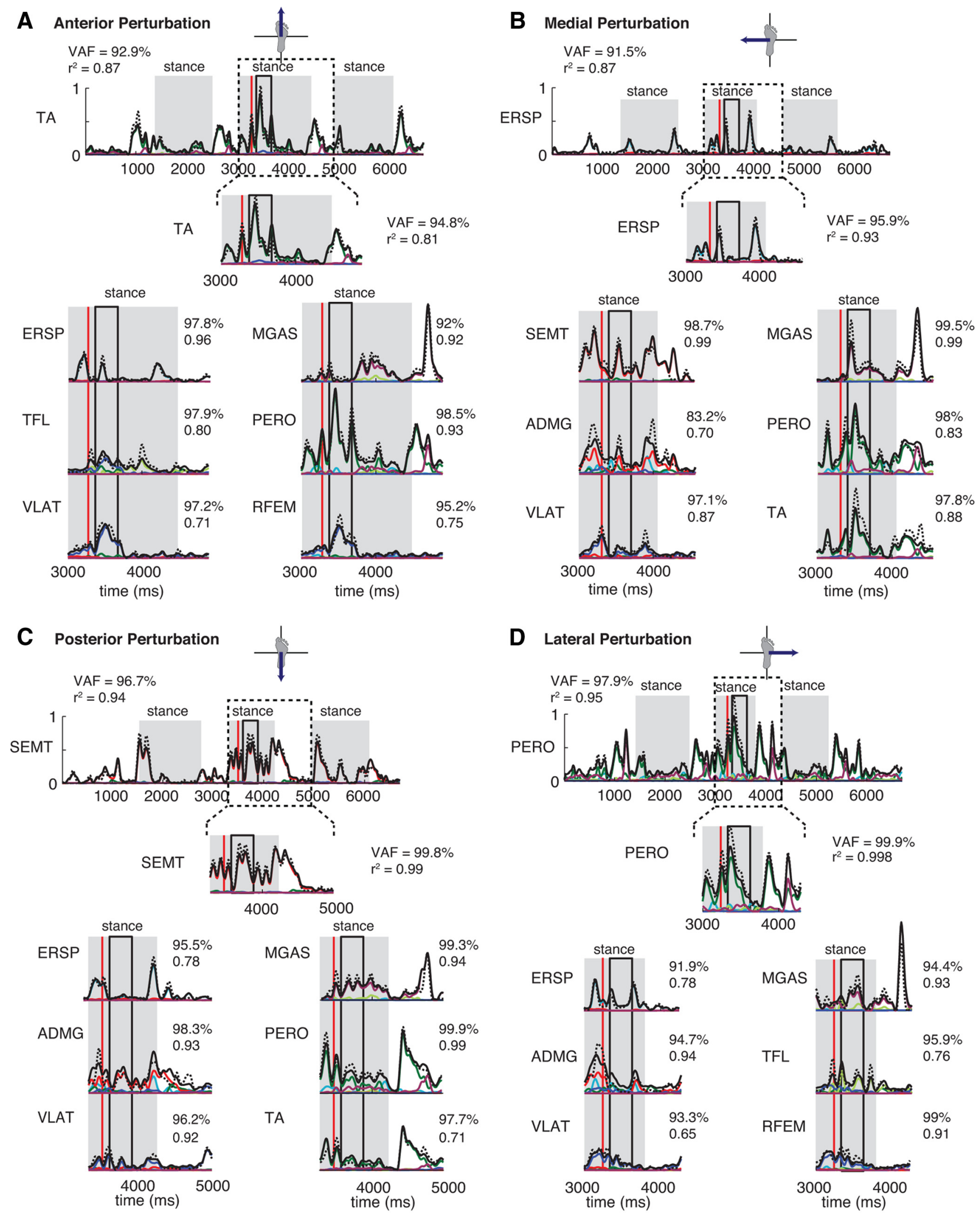

TA
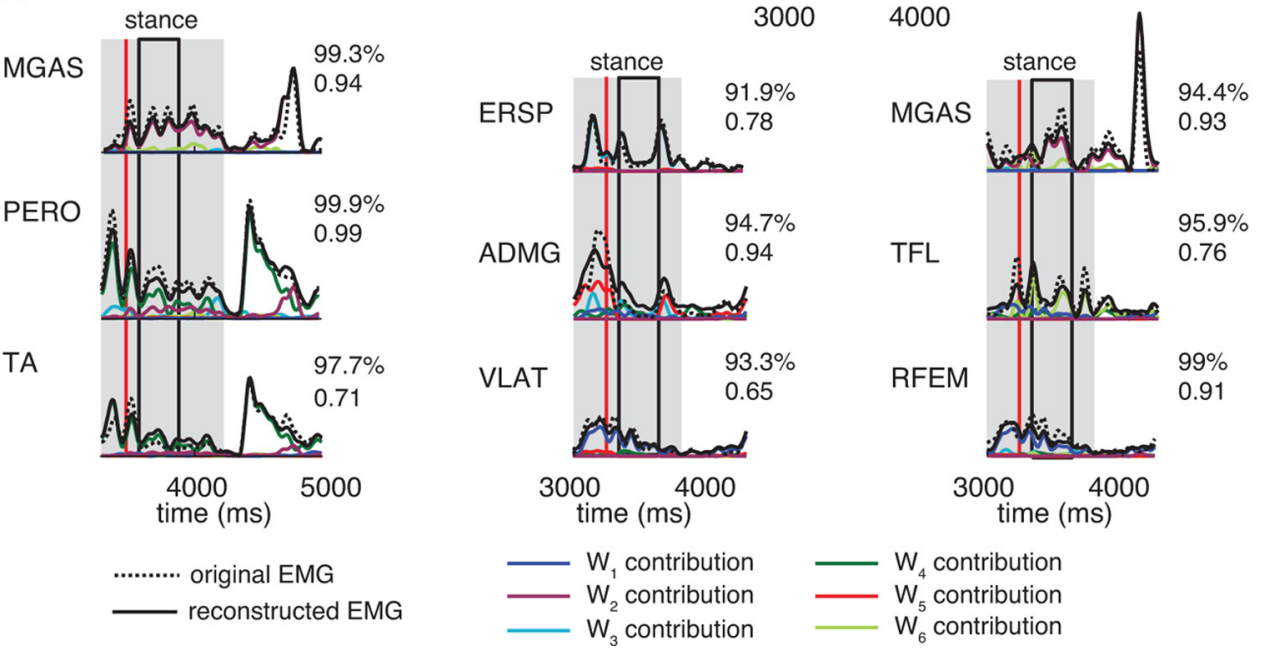

Figure 8. Reconstructions of EMGs in several perturbed walking trials using the muscle synergies shown in Figure 4. Original data are shown by a dashed black line and reconstructed data are shown by a solid black line. The contribution of each muscle synergy to the reconstruction is shown by the corresponding colored line, all of which are added to generate (Figure legend continues.) 
A Lateral Perturbation

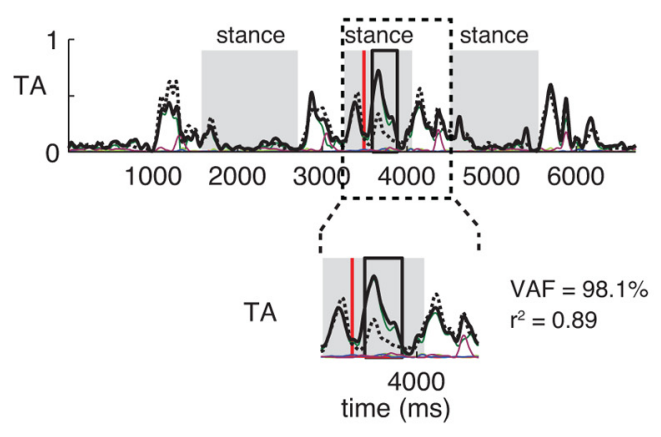

......... original EMG

— reconstructed EMG

$-W_{1}$ contribution
$-W_{2}$ contribution
$W_{3}$ contribution

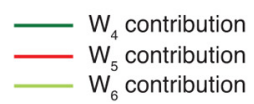

B Anterior Perturbation

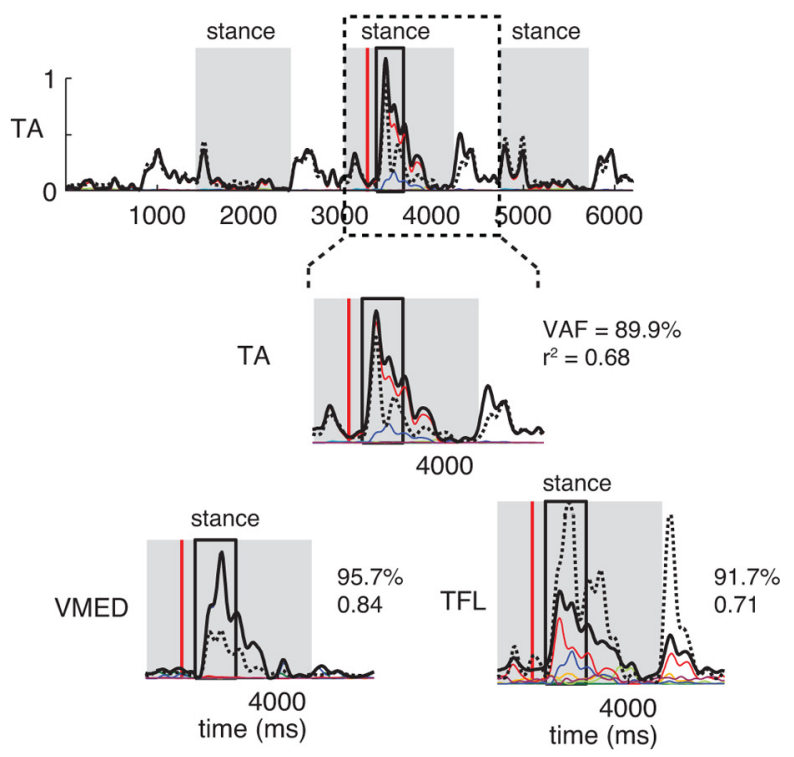

Figure 9. Reconstructions of EMGs using the locomotor muscle synergies for a few trials and muscles that were not well reconstructed (4.2\% of all trials and muscles contained postural responses that were not as well reconstructed using the locomotor muscle synergies). $A$, A lateral perturbation during slow walking in which the postural response in TA was overpredicted by the locomotor muscle synergies shown in Figure 4. Original data are shown by a dashed black line and reconstructed data are shown by a solid black line. The contribution of each muscle synergy to the reconstruction is shown by the corresponding colored line, all of which are added to generate the total reconstruction. $\boldsymbol{B}$, Subject 5; anterior perturbation during walking in which the postural response in TFL was underpredicated by their locomotor muscle synergies, and the postural responses in VMED and TA were overpredicted by the locomotor muscle synergies. Shown are VAF and $r^{2}$ between each EMG and the reconstruction using the locomotor muscle synergies.

sponse $100-400 \mathrm{~ms}$ after perturbation. $\left(\mathrm{VAF}=91 \pm 8.7 \%, r^{2}=\right.$ $0.70 \pm 0.25$, Fig. 8 ). By visual inspection, a few cases ( 142 of 3392 total reconstructions across all subjects and trials, or $4.2 \%$ of all reconstructions) were identified where the fits between the measured muscle activity and the reconstruction using the muscle synergies from walking could be improved; these were found to have significantly lower reconstruction values than the group average: $\mathrm{VAF}=75.2 \pm 18.5 \%\left(p<10^{-16} ; t_{(3532)}=13.1\right)$ and $r^{2}$ $=0.50 \pm 0.24\left(p<10^{-11} ; t_{(3073)}=6.9\right)$. In some cases, muscle activity was overpredicted based on muscle synergy recruitment; for example, in a lateral perturbation TA activity was overpredicted, although the activity of other muscles was well reconstructed (Fig. 9A). Alternately, in the same trial muscle activity for one muscle could be overpredicted (Fig. 9B, VMED and TA), whereas another muscle could be underpredicted (Fig. 9B, TFL).

\section{Discussion}

This is the first demonstration that voluntary and reactive modifications in muscle activity during human walking can be explained by variations in the temporal recruitment of spatially fixed locomotor muscle synergies. Our work corroborates animal studies demonstrating consistent muscle synergy patterns but different recruitment phases in locomotor patterns and voluntary gait modification (Drew et al., 2008), and variable temporal

(Figure legend continued.) the total reconstruction. Average VAF \pm SD between each muscle activation pattern and the muscle synergy reconstruction was $87.8 \pm 8.2 \%$ for the entire trial, and $91 \pm 8.7 \%$ for the perturbation response time window $100-400 \mathrm{~ms}$ after perturbation. $A-D$, Posterior perturbation during slow walking $(A)$, lateral perturbation during slow walking $(B)$, medial perturbation during slow walking $(\boldsymbol{C})$, and anterior perturbation during slow walking (D). Shown are VAF and $r^{2}$ between each EMG and the reconstruction using the locomotor muscle synergies. recruitment of consistent muscle synergies across automatic and reactive motor behaviors in reduced preparations (Kargo and Giszter, 2008; Cheung et al., 2009a; Roh et al., 2011). Together with the results of other studies, these findings suggest that muscle synergies for lower limb tasks may be spatially fixed and encoded in the spinal cord (Hart and Giszter, 2004, 2010; Cheung et al., 2005; Kargo et al., 2010), but recruited in parallel from a variety of pathways (Roh et al., 2011). For robust, natural walking patterns, these would include the central pattern generator for walking, brainstem-mediated pathways used for reactive balance control, and motor cortical pathways for voluntary modifications of gait. Similar convergent pathways exist for the control of the upper limb (Dum and Strick, 1996; Davidson et al., 2007; Takei and Seki, 2010; Alstermark and Isa, 2012), therefore muscle synergies may represent the lowest level of functional organization in the motor repertoire that can be recruited in parallel by different hierarchical neural pathways for voluntary, automatic, and reactive motor behaviors.

The recruitment of a common set of muscle synergies across cycle-by-cycle variations in walking is consistent with neurophysiological evidence that temporal and spatial aspects of locomotor pattern generation are distinct and hierarchically organized. We observed differences in step length, step width, and stance duration in perturbed steps (You, 2001), yet muscle activation patterns were still well accounted for by altering the temporal recruitment of muscle synergies recruited in unperturbed walking. Similarly, variations in muscle activation patterns (Clark et al., 2010) across a wide range of walking speeds have been explained by graded changes in the temporal recruitment of a common set of muscle synergies. Such observations are consistent with neurophysiological studies that demonstrate that temporal rhythm generators in the spinal cord project to spatial 
pattern formation networks that recruit multiple motoneuron pools (Burke et al., 2001; McCrea and Rybak, 2008). In fictive spinal preparations, deletions in muscle activity bursts during a consistent locomotor rhythm provide evidence of this hierarchical arrangement of temporal and spatial pattern formation (Lafreniere-Roula and McCrea, 2005). Similarly, we showed in human walking that some muscle synergies were recruited inconsistently or not at all during slow walking, during which time other muscle synergies displayed regular, phasic modulation (den Otter et al., 2004).

Recruitment of locomotor muscle synergies before perturbations suggests that voluntary modifications to gait from descending pathways act in parallel with subcortical pathways generating the base locomotor pattern. Although anticipation does not affect walking kinematics (Pijnappels et al., 2001), we observed that some locomotor muscle synergies were recruited more strongly in anticipation of a perturbation. In some cases, this anticipatory activity involved recruitment of muscle synergies in atypical phases of gait, suggesting activity of parallel descending pathways separate from those responsible for the base locomotor pattern. Similarly, gait modifications for obstacle avoidance in cats are mediated by pyramidal tract neurons in motor cortex, which alter the magnitude and phase of muscle synergy recruitment but not muscle synergy structure (Drew et al., 2002; Drew et al., 2008). Thus the timing but not spatial structure of muscle activity for gait modification is specified by motor cortical activity, and is independent of generation of the base locomotor pattern. The structure of locomotor muscle synergies may be encoded in subcortical structures that receive convergent descending projections (Rathelot and Strick, 2009; Alstermark and Isa, 2012). Accordingly damage to the cortex due to stroke alters temporal but not spatial aspects of muscle activity during reaching and walking (Cheung et al., 2009b; Clark et al., 2010), such that normally independent muscle synergies are recruited together (Clark et al., 2010).

Recruitment of locomotor muscle synergies for reactive balance control further suggests parallel recruitment of locomotor muscle synergies by sensorimotor feedback pathways mediating balance control. We found that locomotor muscle synergies were recruited in response to perturbations during walking at latencies $(\sim 100 \mathrm{~ms})$ similar to those observed in response to perturbations during standing balance (Horak and Macpherson, 1996; TorresOviedo and Ting, 2010). Such long-latency responses are likely mediated by brainstem pathways for balance control (Macpherson and Fung, 1999; Deliagina et al., 2008). Evidence in cats demonstrates that reticulospinal neurons mediate reactive as well as voluntary balance control independent of the muscle or even limbs that are recruited (Schepens and Drew, 2004; Schepens et al., 2008). Thus it is likely that brainstem neurons specify temporal but not spatial patterns of muscle activity, recruiting downstream muscle synergies to implement the muscle coordination patterns for balance (Misiaszek, 2003). Similarly, in patients with spinal cord injury, responses to perturbations during walking appear to be organized at the spinal level and modulated by supraspinal centers (Field-Fote and Dietz, 2007). Our results suggest that muscle synergies for locomotion receive convergent descending inputs that specify the magnitude and timing of recruitment for balance and locomotion. However, further study is required to evaluate whether the muscle synergies identified during walking are similar to those evoked during perturbations to standing balance (Torres-Oviedo and Ting, 2007).

The recruitment of muscle synergies by parallel descending pathways may reflect a general principle for motor control in both the upper and lower extremities, such that muscle synergies provide a motor repertoire, or library of motor subtasks that can be flexibly recruited to generate a variety of complex natural movements (Giszter et al., 2007; Ting, 2007; Tresch and Jarc, 2009). In reaching and grasp (Gentner and Classen, 2006; Overduin et al., 2008; Gentner et al., 2010; d'Avella et al., 2011), muscle synergy activity is thought to reflect the activity of cortico-motoneuronal (CM) cells (Holdefer and Miller, 2002), which can project to multiple motoneurons. CM cells can also project to reticulospinal (Davidson et al., 2007) and propriospinal interneurons (Rathelot and Strick, 2009; Alstermark and Isa, 2012), which in turn have divergent projections to multiple motoneurons. Thus there are many possible neural substrates for the consistent spatial muscle synergy structure observed across a variety of motor tasks (Drew et al., 2008; Overduin et al., 2008; Torres-Oviedo and Ting, 2010; Chvatal et al., 2011; Roh et al., 2012).

Thus, muscle synergies may represent the lowest level of the motor control hierarchy, recruited by various pathways to achieve task-level goals. Muscle synergies may provide a mechanism by which task-level goals are translated into complex execution-level patterns of muscle activity, consistent with the representation of abstract motor variables throughout the neuraxis, including the cortex (Moran and Schwartz, 1999; Schwartz and Moran, 2000), superior colliculus (Stuphorn et al., 2000), and spinal cord (Bosco and Poppele, 2001). Accordingly muscle synergies in locomotion and balance have been demonstrated to produce consistent biomechanical functions (Raasch and Zajac, 1999; Ting and Macpherson, 2005; McKay and Ting, 2008; Neptune et al., 2009; Chvatal et al., 2011). Thus, the modular control of muscles may reflect a repertoire of motor actions, accessible by multiple motor pathways to achieve abstract motor goals that are independent of joint motions (Frey et al., 2011), such as hand movement direction in reaching (Georgopoulos et al., 1986; Scott and Kalaska, 1997; d'Avella et al., 2011), force generation (Roh et al., 2012), and center of mass movement direction in balance (Torres-Oviedo et al., 2006; Chvatal et al., 2011; Safavynia and Ting, 2012).

\section{References}

Alstermark B, Isa T (2012) Circuits for skilled reaching and grasping. Annu Rev Neurosci 35:559-578.

Bachmann V, Müller R, van Hedel HJ, Dietz V (2008) Vertical perturbations of human gait: organisation and adaptation of leg muscle responses. Exp Brain Res 186:123-130.

Bhatt T, Wening JD, Pai YC (2006) Adaptive control of gait stability in reducing slip-related backward loss of balance. Exp Brain Res 170:61-73.

Bosco G, Poppele RE (2001) Proprioception from a spinocerebellar perspective. Physiol Rev 81:539-568.

Burke RE, Degtyarenko AM, Simon ES (2001) Patterns of locomotor drive to motoneurons and last-order interneurons: clues to the structure of the CPG. J Neurophysiol 86:447-462.

Cheung VC, d'Avella A, Tresch MC, Bizzi E (2005) Central and sensory contributions to the activation and organization of muscle synergies during natural motor behaviors. J Neurosci 25:6419-6434.

Cheung VC, d'Avella A, Bizzi E (2009a) Adjustments of motor pattern for load compensation via modulated activations of muscle synergies during natural behaviors. J Neurophysiol 101:1235-1257.

Cheung VC, Piron L, Agostini M, Silvoni S, Turolla A, Bizzi E (2009b) Stability of muscle synergies for voluntary actions after cortical stroke in humans. Proc Natl Acad Sci U S A 106:19563-19568.

Chvatal SA, Torres-Oviedo G, Safavynia SA, Ting LH (2011) Common muscle synergies for control of center of mass and force in nonstepping and stepping postural behaviors. J Neurophysiol 106:999-1015.

Clark DJ, Ting LH, Zajac FE, Neptune RR, Kautz SA (2010) Merging of healthy motor modules predicts reduced locomotor performance and muscle coordination complexity post-stroke. J Neurophysiol 103:844-857. 
d'Avella A, Portone A, Fernandez L, Lacquaniti F (2006) Control of fastreaching movements by muscle synergy combinations. J Neurosci 26:7791-7810.

d'Avella A, Portone A, Lacquaniti F (2011) Superposition and modulation of muscle synergies for reaching in response to a change in target location. J Neurophysiol 106:2796-2812.

Davidson AG, Schieber MH, Buford JA (2007) Bilateral spike-triggered average effects in arm and shoulder muscles from the monkey pontomedullary reticular formation. J Neurosci 27:8053-8058.

Deliagina TG, Beloozerova IN, Zelenin PV, Orlovsky GN (2008) Spinal and supraspinal postural networks. Brain Res Rev 57:212-221.

den Otter AR, Geurts AC, Mulder T, Duysens J (2004) Speed related changes in muscle activity from normal to very slow walking speeds. Gait Posture 19:270-278.

Drew T (1988) Motor cortical cell discharge during voluntary gait modification. Brain Res 457:181-187.

Drew T, Jiang W, Widajewicz W (2002) Contributions of the motor cortex to the control of the hindlimbs during locomotion in the cat. Brain Res Brain Res Rev 40:178-191.

Drew T, Kalaska J, Krouchev N (2008) Muscle synergies during locomotion in the cat: a model for motor cortex control. J Physiol 586:1239-1245.

Dum RP, Strick PL (1996) Spinal cord terminations of the medial wall motor areas in macaque monkeys. J Neurosci 16:6513-6525.

Field-Fote EC, Dietz V (2007) Single joint perturbation during gait: preserved compensatory response pattern in spinal cord injured subjects. Clin Neurophysiol 118:1607-1616.

Forssberg H, Grillner S, Halbertsma J, Rossignol S (1980) The locomotion of the low spinal cat. II. Interlimb coordination. Acta Physiol Scand 108:283-295.

Frey SH, Fogassi L, Grafton S, Picard N, Rothwell JC, Schweighofer N, Corbetta M, Fitzpatrick SM (2011) Neurological principles and rehabilitation of action disorders: computation, anatomy, and physiology (CAP) model. Neurorehabil Neural Repair 25:6S-20S.

Gentner R, Classen J (2006) Modular organization of finger movements by the human central nervous system. Neuron 52:731-742.

Gentner R, Gorges S, Weise D, aufm Kampe K, Buttmann M, Classen J (2010) Encoding of motor skill in the corticomuscular system of musicians. Curr Biol 20:1869-1874

Georgopoulos AP, Schwartz AB, Kettner RE (1986) Neuronal population coding of movement direction. Science 233:1416-1419.

Giszter S, Patil V, Hart C (2007) Primitives, premotor drives, and pattern generation: a combined computational and neuroethological perspective. Prog Brain Res 165:323-346.

Gorassini MA, Prochazka A, Hiebert GW, Gauthier MJ (1994) Corrective responses to loss of ground support during walking. I. Intact cats. J Neurophysiol 71:603-610.

Hart CB, Giszter SF (2004) Modular premotor drives and unit bursts as primitives for frog motor behaviors. J Neurosci 24:5269-5282.

Hart CB, Giszter SF (2010) A neural basis for motor primitives in the spinal cord. J Neurosci 30:1322-1336.

Henry SM, Fung J, Horak FB (1998) EMG responses to maintain stance during multidirectional surface translations. J Neurophysiol 80: $1939-1950$.

Herter TM, Korbel T, Scott SH (2009) Comparison of neural responses in primary motor cortex to transient and continuous loads during posture. J Neurophysiol 101:150-163.

Hiebert GW, Gorassini MA, Jiang W, Prochazka A, Pearson KG (1994) Corrective responses to loss of ground support during walking. II. Comparison of intact and chronic spinal cats. J Neurophysiol 71:611-622.

Hof AL, Elzinga H, Grimmius W, Halbertsma JP (2002) Speed dependence of averaged EMG profiles in walking. Gait Posture 16:78-86.

Holdefer RN, Miller LE (2002) Primary motor cortical neurons encode functional muscle synergies. Exp Brain Res 146:233-243.

Honeycutt CF, Gottschall JS, Nichols TR (2009) Electromyographic responses from the hindlimb muscles of the decerebrate cat to horizontal support surface perturbations. J Neurophysiol 101:2751-2761.

Horak FB, Macpherson JM (1996) Postural orientation and equilibrium. In: Handbook of physiology, Section 12, pp 255-292. New York: American Physiological Society.

Isa T, Ohki Y, Alstermark B, Pettersson LG, Sasaki S (2007) Direct and indirect cortico-motoneuronal pathways and control of hand/arm movements. Physiology (Bethesda) 22:145-152.
Ivanenko YP, Poppele RE, Lacquaniti F (2004) Five basic muscle activation patterns account for muscle activity during human locomotion. J Physiol 556:267-282.

Kargo WJ, Giszter SF (2008) Individual premotor drive pulses, not timevarying synergies, are the units of adjustment for limb trajectories constructed in spinal cord. J Neurosci 28:2409-2425.

Kargo WJ, Ramakrishnan A, Hart CB, Rome LC, Giszter SF (2010) A simple experimentally based model using proprioceptive regulation of motor primitives captures adjusted trajectory formation in spinal frogs. J Neurophysiol 103:573-590.

Lafreniere-Roula M, McCrea DA (2005) Deletions of rhythmic motoneuron activity during fictive locomotion and scratch provide clues to the organization of the mammalian central pattern generator. J Neurophysiol 94:1120-1132.

Lee DD, Seung HS (1999) Learning the parts of objects by non-negative matrix factorization. Nature 401:788-791.

Macpherson JM, Fung J (1999) Weight support and balance during perturbed stance in the chronic spinal cat. J Neurophysiol 82:3066-3081.

Maki BE, McIlroy WE, Perry SD (1996) Influence of lateral destabilization on compensatory stepping responses. J Biomech 29:343-353.

McCrea DA, Rybak IA (2008) Organization of mammalian locomotor rhythm and pattern generation. Brain Res Rev 57:134-146.

McKay JL, Ting LH (2008) Functional muscle synergies constrain force production during postural tasks. J Biomech 41:299-306.

Misiaszek JE (2003) Early activation of arm and leg muscles following pulls to the waist during walking. Exp Brain Res 151:318-329.

Moran DW, Schwartz AB (1999) Motor cortical representation of speed and direction during reaching. J Neurophysiol 82:2676-2692.

Neptune RR, Clark DJ, Kautz SA (2009) Modular control of human walking: a simulation study. J Biomech 42:1282-1287.

Oddsson LI, Wall C, McPartland MD, Krebs DE, Tucker CA (2004) Recovery from perturbations during paced walking. Gait Posture 19:24-34.

Overduin SA, d'Avella A, Roh J, Bizzi E (2008) Modulation of muscle synergy recruitment in primate grasping. J Neurosci 28:880-892.

Pijnappels M, Bobbert MF, van Dieën JH (2001) Changes in walking pattern caused by the possibility of a tripping reaction. Gait Posture 14:11-18.

Raasch CC, Zajac FE (1999) Locomotor strategy for pedaling: muscle groups and biomechanical functions. J Neurophysiol 82:515-525.

Rathelot JA, Strick PL (2009) Subdivisions of primary motor cortex based on cortico-motoneuronal cells. Proc Natl Acad Sci U S A 106:918-923.

Roh J, Cheung VC, Bizzi E (2011) Modules in the brain stem and spinal cord underlying motor behaviors. J Neurophysiol 106:1363-1378.

Roh J, Rymer WZ, Beer RF (2012) Robustness of muscle synergies underlying three-dimensional force generation at the hand in healthy humans. J Neurophysiol 107:2123-2142.

Rossignol S, Dubuc R, Gossard JP (2006) Dynamic sensorimotor interactions in locomotion. Physiol Rev 86:89-154.

Rossignol S, Barrière G, Frigon A, Barthélemy D, Bouyer L, Provencher J, Leblond H, Bernard G (2008) Plasticity of locomotor sensorimotor interactions after peripheral and/or spinal lesions. Brain Res Rev $57: 228-240$.

Safavynia SA, Ting LH (2012) Task-level feedback can explain temporal recruitment of spatially-fixed muscle synergies throughout postural perturbations. J Neurophysiol 107:159-177.

Schepens B, Drew T (2004) Independent and convergent signals from the pontomedullary reticular formation contribute to the control of posture and movement during reaching in the cat. J Neurophysiol 92:2217-2238.

Schepens B, Stapley P, Drew T (2008) Neurons in the pontomedullary reticular formation signal posture and movement both as an integrated behavior and independently. J Neurophysiol 100:2235-2253.

Schwartz AB, Moran DW (2000) Arm trajectory and representation of movement processing in motor cortical activity. Eur J Neurosci 12:1851-1856.

Scott SH, Kalaska JF (1997) Reaching movements with similar hand paths but different arm orientations. I. Activity of individual cells in motor cortex. J Neurophysiol 77:826-852.

Studenski S, Perera S, Wallace D, Chandler JM, Duncan PW, Rooney E, Fox M, Guralnik JM (2003) Physical performance measures in the clinical setting. J Am Geriatr Soc 51:314-322.

Stuphorn V, Bauswein E, Hoffmann KP (2000) Neurons in the primate superior colliculus coding for arm movements in gaze-related coordinates. J Neurophysiol 83:1283-1299. 
Takei T, Seki K (2010) Spinal interneurons facilitate coactivation of hand muscles during a precision grip task in monkeys. J Neurosci 30:17041-17050.

Tang PF, Woollacott MH, Chong RK (1998) Control of reactive balance adjustments in perturbed human walking: roles of proximal and distal postural muscle activity. Exp Brain Res 119:141-152.

Ting LH (2007) Dimensional reduction in sensorimotor systems: a framework for understanding muscle coordination of posture. Prog Brain Res 165:299-321.

Ting LH, Chvatal SA (2010) Decomposing muscle activity in motor tasks: methods and interpretation. In: Motor control: theories, experiments, and applications (Danion F, Latash M, eds). New York: Oxford UP.

Ting LH, Macpherson JM (2005) A limited set of muscle synergies for force control during a postural task. J Neurophysiol 93:609-613.

Ting LH, McKay JL (2007) Neuromechanics of muscle synergies for posture and movement. Curr Opin Neurobiol 17:622-628.

Ting LH, Kautz SA, Brown DA, Zajac FE (1999) Phase reversal of biomechanical functions and muscle activity in backward pedaling. J Neurophysiol 81:544-551.

Torres-Oviedo G, Ting LH (2007) Muscle synergies characterizing human postural responses. J Neurophysiol 98:2144-2156.

Torres-Oviedo G, Ting LH (2010) Subject-specific muscle synergies in hu- man balance control are consistent across different biomechanical contexts. J Neurophysiol 103:3084-3098.

Torres-Oviedo G, Macpherson JM, Ting LH (2006) Muscle synergy organization is robust across a variety of postural perturbations. J Neurophysiol 96:1530-1546.

Tresch MC, Jarc A (2009) The case for and against muscle synergies. Curr Opin Neurobiol 19:601-607.

Tresch MC, Saltiel P, Bizzi E (1999) The construction of movement by the spinal cord. Nat Neurosci 2:162-167.

Tresch MC, Cheung VC, d'Avella A (2006) Matrix factorization algorithms for the identification of muscle synergies: evaluation on simulated and experimental data sets. J Neurophysiol 95:2199-2212.

Welch TJ, Ting LH (2009) A feedback model explains the differential scaling of human postural responses to perturbation acceleration and velocity. J Neurophysiol 101:3294-3309.

Winter DA, Yack HJ (1987) EMG profiles during normal human walking: stride-to-stride and inter-subject variability. Electroencephalogr Clin Neurophysiol 67:402-411.

You J, Chou Y, Lin C, Su F (2001) Effect of slip on movement of body center of mass relative to base of support. Clin Biomech 16:167-173.

Zar J (1999) Biostatistical analysis. Upper Saddle River, NJ: Prentice-Hall. 